\title{
A Comparison of Raman and EDXRF Chemical Imaging for Use in Formulation Process Development and Quality Control
}

\author{
Fiona Clarke*, Andrew Whitley**, Sergey Mamedov**, Fran Adar**, Neil Lewis***, and Eunah \\ Lee*** \\ *Pfizer, Process Analytical Support Group, Sandwich Laboratories, Kent CT13 9NJ, UK \\ **Jobin Yvon Horiba, 3880 Park Ave. Edison, NJ 08820 \\ ***Spectral Dimensions, Inc., 3416 Olandwood Court, Olney, MD 20832
}

Dispersion of the API (active pharmaceutical ingredient) and excipients in pharmaceutical tablets is of vital importance to the final product. Among other things the components' particle sizes and distribution can determine correct dosage as well as correct chemical behavior between the components during shelf life and during ingestion.

Compounds of magnesium and calcium are common components of pharmaceutical formulations. Spectroscopic imaging, and in particular hyper-spectral imaging, can provide a complete understanding of a formulation. One of the excipients, magnesium stearate, which is often added because of its lubricating properties, can be difficult to observe visually. In this paper we will compare two spectral imaging techniques - Raman Microscopy and Energy Dispersive X-Ray Fluorescence (EDXRF) Microscopy. These two techniques, Raman and EDXRF microscopy, provide molecular and elemental images respectively. Both analytical techniques are being used in process development and quality control. Data will be shown that highlights the capabilities of both techniques and discusses the advantages of each. Raman microscopy allows very high spatial resolution of molecular maps $(1 \mu \mathrm{m})$; hyperspectral images very accurately show particle size and distribution in a formulation. The EDXRF microscope provides a rapid method to obtain hyperspectral elemental images with $10 \mu \mathrm{m}$ spatial resolution. For both techniques little or no sample preparation is required.

The simplest method of preparing chemical maps is to bracket peaks of the species of interest and then color-map the intensity at each pixel of the various species. This works well as long as each species has a spectral band that does not overlap with bands of other species. The reality is that for complex organic species this requirement is often not met in Raman multifiles. In such cases treatment of the spectra with multivariate techniques enables one to separate the variances of the individual species. In addition, this can be helpful in EDXRF as well, even though the spectra are much simpler, because of the potential for overlap between elemental lines.

Figure 1 shows the Raman spectra of four excipients plus the API in a pharmaceutical product. Raman images produced by bracketing the bands of microcrystalline cellulose (MCC), dicalcium phosphate (DCP), and sodium starch glycolate (SSG) are shown as an RGB micrograph. The map of magnesium stearate is produced using multivariate algorithms of ISys ${ }^{\mathrm{TM}}$. In addition to producing such intensity maps, the software enables one to perform statistics on the multifile, to set thresholds in the maps, and to calculate the percentage of area that is occupied by the species of interest. This capability then allows particle size and morphology and concentrations to be estimated. 
Figure 2 shows EDXRF maps of $\mathrm{Ca}, \mathrm{Mg}$, and $\mathrm{P}$. The $\mathrm{Mg}$ is fairly homogeneous, consistent with its known properties. Clear correlations between $\mathrm{Ca}$ and $\mathrm{P}$ are seen, as expected because of the presence of a calcium phosphate phase.
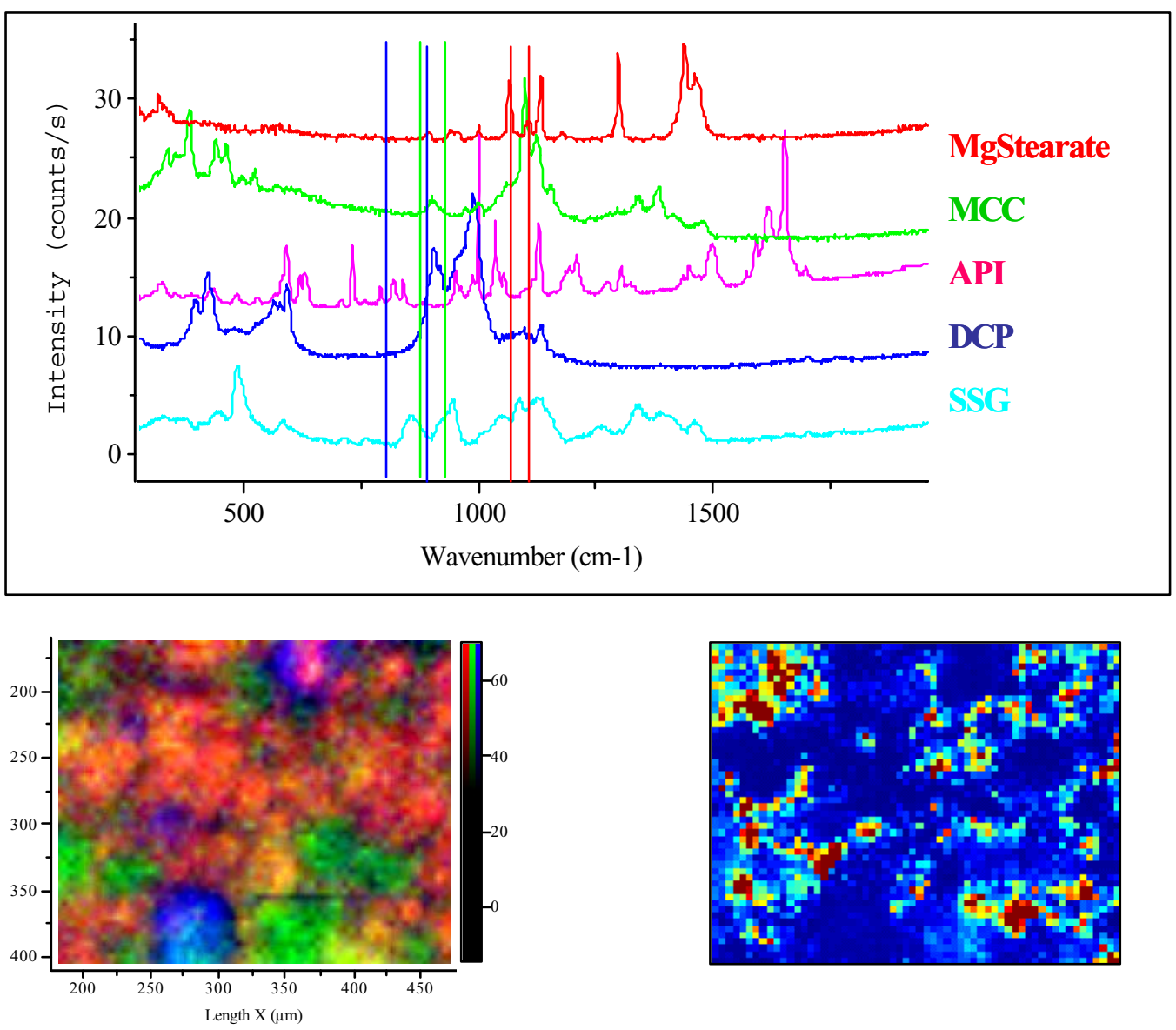

Fig.1. Top: Raman spectra of 4 excipients plus API. 3 sets of vertical lines indicate the spectral line used to produce the RGB color coded map on the bottom left. Bottom right: Intensity map of magnesium stearate produced from the multivariate factor corresponding to this component.

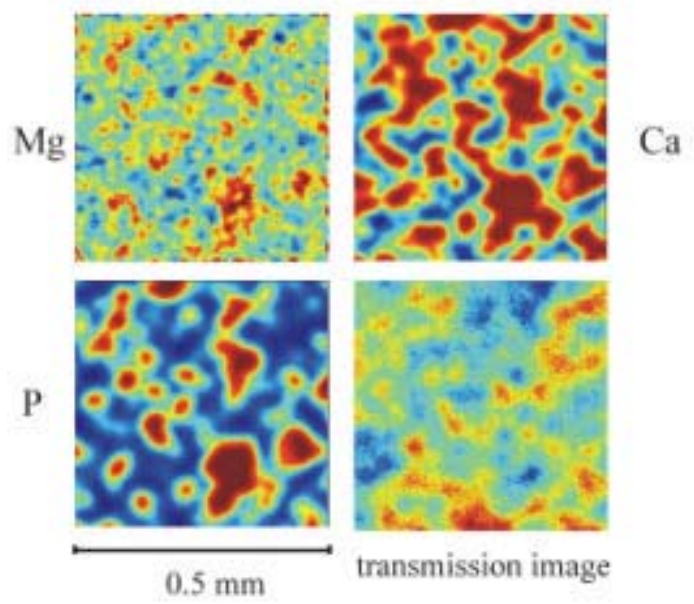

Fig. 2. EDXRF map of $\mathrm{Mg}, \mathrm{Ca}$ and $\mathrm{P}$ and the $\mathrm{X}$-ray transmission image acquired with the $10 \mu \mathrm{m}$ capillary. 